\title{
Sertraline improves depression scores in the elderly in the short term, regardless of medical comorbidity status
}

Sheikh Jl, Cassidy EL, Doraiswamy PM, et al. Efficacy, safety, and tolerability of sertraline in patients with late-life depression and comorbid medical illness. J Am Geriatr Soc 2004;52:86-92.

\section{What are the effects of sertraline in elderly people with depression, taking comorbid medical illnesses into consideration?}

\section{METHODS}

$\square$

Design: Randomised, double blind, placebo controlled trial.

$\gg$

Allocation: Concealed.

Blinding: Participants and assessors blinded.

Follow up period: Eight weeks with assessments at baseline and endpoint; the three primary outcome variables were also assessed every 2 weeks.

Setting: Multicentre trial in the USA; timeframe not specified.

Patients: 752 people, age $\geqslant 60$ years $(75 \% \geqslant 65)$ with major depressive disorder (MDD; DSM-IV criteria), and scoring $\geqslant 18$ on the Hamilton Depression Scale (HAMD) with a score $\geqslant 2$ on item 1 (depressed mood), and a minimum of 4 weeks of symptoms. Exclusions: bipolar disorder, schizophrenia, or other psychosis; Mini-Mental State Exam (MMSE) score <24; DSM-IV diagnosis of dementia, organic mental disorder, or mental retardation; or a significant unstable medical condition.

$\mathbf{R}_{\mathbf{X}}$

Intervention: Sertraline 50-100 mg, flexible dose; placebo for 8 weeks.

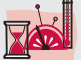

Outcomes: Depression, measured with the primary outcomes: HAMD; Clinical Global Impression-Severity scale (CGI-S) and the Clinical Global Impression-Improvement (CGI-I) scale. Response was defined as an endpoint CGI-I score of 1 (very much) or 2 (much) and a reduction $\geqslant 50 \%$ in HAMD. Safety and adverse events also assessed.

Patient follow up: $97 \%$ of participants received at least one dose of study medication and were included in the ITT analysis. Safety: data presented for $80 \%$ of participants.

\section{MAIN RESULTS}

The intention to treat analysis included 728 participants. 442 participants had medical comorbiditities (vascular disease, diabetes, or arthritis) and were likely to be older, retired, widowed, female, and to have lower ratings of quality of life and functioning than those with no comorbidities $(n=127)$. Efficacy: at 8 weeks, sertraline significantly improved depression scores compared with placebo for the overall sample (see http://www.ebmentalhealth.com/supplemental for table). There were no significant differences in changes of depression score from baseline between people with and without comorbidities for both the placebo and sertraline groups. Adverse events: data presented for 458 participants. More people taking sertraline discontinued the trial compared with placebo; however statistical comparisons between groups are not presented (sertraline: $25 / 172,15 \% \vee$ placebo $11 / 232,5 \%)$. Discontinuation rates were

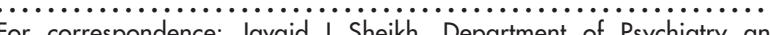
For correspondence: Javaid I Sheikh, Department of Psychiatry and Behavioral Sciences, Stanford University School of Medicine, 401 Quarry Road, Stanford, CA, USA, 94305; sheikh@stanford.edu

Sources of funding: Pfizer Pharmaceuticals. similar for people with and without comorbidities for both the sertraline and placebo groups.

\section{CONCLUSIONS}

Sertraline improved depression scores in elderly people both with and without comorbidities.

\section{NOTES}

Results from the 159 people without vascular disease, diabetes, or arthritis but with a current prescription or hospitalisation in the preceding year are not reported in this study. To be included in the ITT analysis, participants only had to take one dose of medication. More detailed information on the adherence rate, or number of doses taken by participants are not presented. Discontinuation rates are presented for $458 / 569$ (80\%) participants.

\section{Commentary}

- o far, studies on treatment of depression in the elderly have generally included only people in good physical health, thereby excluding and neglecting somatic comorbidity, which is very prevalent in such patients. This large, placebo controlled study considers older age as well as somatic comorbidity and provides important information on how to treat this specific group of patients.

The positive findings about efficacy and tolerability of sertraline are encouraging, and consistent with the results of our prospective postmarketing surveillance study on serotonin reuptake inhibitors in late life depression. ' As it is well recognised that the prevalence of this disorder amounts to $12 \%-15 \%^{2}$ and that elderly patients are mostly unable to tolerate tricyclic antidepressants, SSRIs are a promising first choice in the treatment of late life depression.

It is important to recognise that the mean daily sertraline dosage is quite similar between various comorbidity groups. Sheik et al also showed that elderly comorbid patients tolerate sertraline in a dosage within the therapeutic range.

The fact that sertraline treated patients with medical comorbidity had consistently lower rates of adverse events may appear counterintuitive at first glance. On the other hand, considering that many reported side effects of sertraline are somatic in nature, one might speculate that people with stable comorbidity develop an increasing tolerance to physical symptoms simply because they have gotten used to different kinds of somatic problems.

The authors also show the importance of quality of life (QOL) measures in such studies. Impaired QOL is common in people with late life depression and is associated with somatic comorbidity. Unfortunately, they only provide baseline $\mathrm{QOL}$ information and do not report QOL changes over the course of the study.

As of now, studies on this topic were not designed to study elderly depressed patients with serious medical illness and did not quantify medical comorbidity. In addition, they generally tended to exclude a variety of concomitant medication and therefore were not representative for many elderly depressive patients presenting for treatment in everyday clinical practice. Therefore this report closes a highly clinically relevant gap in the quest for evidence based treatment for late life depression.

Ilsemarie Kurzthaler, MD, W Wolfgang Fleischhacker, MD Department of Biological Psychiatry, Medical University Innsbruck, Innsbruck, Austria

1 Kurzthaler I, Hotter A, Kemmler G, et al. Risk profile of SSRIs in elderly depressive patients with co-morbid physical illness. Pharmacopsychiatry 2001;34:114-18.

2 Katonia CLE. Depression in old age. Chichester: Wiley, 1994:29-41. 